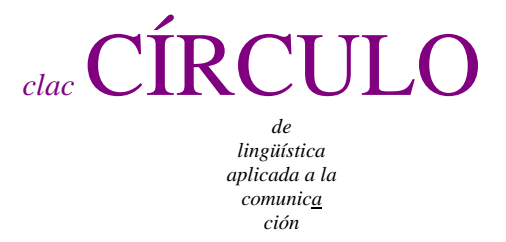

$55 / 2013$

\title{
PERSPECTIVAS EN EL ANÁLISIS DE LA RELACIÓN ENTRE GRAMÁTICA Y DISCURSO: ESTRUCTURA PERIFÉRICA Y CONEXIONES ENTRE ORACIONES
}

\author{
Teresa Rodríguez Ramalle \\ Universidad Complutense de Madrid \\ teresaramalle en ccinf ucm es
}

Resumen

Este número especial, dedicado a las relaciones entre gramática y discurso, muestra un conjunto de trabajos que contribuyen a ofrecernos una perspectiva gramatical de esta relación, basada en el estudio de las diferentes estructuras lingüísticas que se proyectan en la oración.

Palabras clave: gramática, discurso, periferia oracional, estructura informativa, futuro, interpretación concesiva.

Teresa Rodríguez Ramalle. 2013.

Perspectivas en el análisis de la relación entre gramática y discurso: Estructura periférica y conexiones entre oraciones. Círculo de Lingüística Aplicada a la Comunicación 55, 3-8. http://www.ucm.es/info/circulo/no55/ramalle.pdf http://revistas.ucm.es/index.php/CLAC DOI http://dx.doi.org/10.5209/rev_CLAC.2013.v55.43263

(C)2013 Teresa Rodríguez Ramalle Círculo de Lingüística Aplicada a la Comunicación (clac)

Universidad Complutense de Madrid. ISSN 1576-4737. http://www.ucm.es/info/circulo 


\begin{abstract}
This special issue on grammar and discourse shows different articles that offer a grammatical point of view of the relation between grammar and discourse, based on the analysis of different linguistic structures.
\end{abstract}

Key words: grammar, discourse, sentence periphery, informative structure, future, concessive interpretation.

Índice

1. Rasgos gramaticales y periferia 4

2. La relación entre oraciones 5

3. Conclusión 7

Bibliografía 7

\title{
1. Rasgos gramaticales y periferia
}

Los trabajos que se reúnen en este primer número monográfico dedicado a la relación entre gramática y discurso tienen como denominador común el análisis de esta conexión desde un enfoque básicamente gramatical ${ }^{*}$. El significado concreto de este calificativo se podrá encontrar a lo largo de la lectura de los diferentes artículos seleccionados; en todos ellos, las relaciones entre oraciones, ciertas estructuras en la periferia oracional y determinados valores asociados a la subordinación, como la concesividad, se estudian a partir de las marcas lingüísticas concretas que se proyectan en la oración. Aunque los rasgos gramaticales que se van a analizar en este número son

\footnotetext{
* El número monográfico ha sido realizado dentro de la investigación del proyecto GRAMDIS (FFI201020862), financiado por el Ministerio de Economía y Competitividad.
} 
muy diversos: anáforas de diferentes tipos, repeticiones, estructuras parentéticas, tiempos verbales y estructuras de grado, entre otros, todos comparten su dimensión discursiva, pues sirven para establecer conexiones bien entre una oración y la precedente bien entre una oración y las inferencias a las que remite.

Partiendo del estudio de la periferia oracional, el discurso se entiende no como una simple sucesión de oraciones dispuestas de una manera más o menos independiente, sino como un todo integrado, en el que cada constituyente se interpreta teniendo en cuenta tanto su propia estructura sintáctica y semántica como la estructura que representa el contexto de las oraciones precedentes. Según este enfoque, la existencia de conjunciones y partículas discursivas, la estructura informativa de la oración (Smith 2003, Hidalgo Downing 2003), recursos tales como las anáforas asociativas (Garrido 2003) y léxicas (González Ruiz 2008 y Llamas Saiz 2010) contribuyen al establecimiento de unas conexiones oracionales que va más allá de los límites marcados por la subordinación, pues las relaciones también se establecen entre oraciones independientes a partir de mecanismos similares (Asher y Lascarides 2003, Taboada 2006, Garrido 2013, Garrido y Rodríguez Ramalle 2013). El análisis que nos plantea Duque de la estructura textual del discurso electoral de Esperanza Aguirre durante las elecciones autonómicas de 2003 parte de una estructura jerárquica apoyada en las relaciones de coherencia (Mann y Thompson 1988) y en la que los componentes mantienen relaciones semánticas entre sí, que en ocasiones conectan estructuras no necesariamente adyacentes. Este tipo de relaciones se apoya en la presencia de marcas tales como repeticiones y anáforas tanto léxicas como gramaticales.

\section{La relación entre oraciones}

La integración de oraciones dentro de un discurso se hace explícita a través de diferentes mecanismos. Toda construcción requiere y emplea información adicional que no está explicitada en sus constituyentes, como se señala en Lascarides, Copestake y Briscoe (1996). Este tipo de información combina los datos del contexto con los conocimientos que los hablantes poseen acerca de la realidad que comparten y de la que 
hablan. El contexto entendido no solo como la relación que establece una oración con el resto de oraciones dentro de un discurso sino también como los conocimientos compartidos por los hablantes es fundamental para interpretar los vínculos entre oraciones. Tanto los conocimientos previos y compartidos así como los participantes del discurso aparecen codificados en determinadas estructuras sintácticas (Rodríguez Ramalle 2008). La periferia oracional es la estructura donde se integran las relaciones tanto entre oraciones como entre oraciones y conocimientos previos. La inclusión de la presencia del hablante dentro de la periferia permite abrir los estudios lingüísticos a la metodología pragmática, tal y como propone Fuentes Rodríguez en su trabajo. La autora aborda el estudio de la periferia oracional desde una perspectiva que defiende una sintaxis del enunciado, lo que le lleva a reflexionar sobre los límites de la gramática y su extensión al discurso. Para ello se analizan tres tipos de estructuras que tienen en común la subjetividad del hablante: las oraciones comentario, los hedging y los parentéticos.

Un análisis discursivo de estructuras sintácticas relacionadas también con la presencia del hablante es el que nos ofrece Rodríguez Rosique, en su análisis del futuro como una forma que participa en la estrategia contraargumentativa. Para explicar los usos concesivos del futuro, la autora nos plantea una propuesta en la que el futuro debilita la fuerza argumentativa del primer miembro conectado mediante pero, a través de un proceso de distanciamiento por parte del hablante.

Como hemos dicho, el desarrollo de la subordinación y sobre todo de la interpretación de las conexiones de dos estructuras conectadas por medios diversos es una de las líneas de estudio del discurso. Marcovecchio y Pacagnini muestran la relación que existe entre la cuantificación y el desarrollo de valores de contraposición, condicionales y concesivos en las oraciones con para seguidas de infinitivo. Según muestran las autores, del valor básico de finalidad o propósito se desarrollan otros valores vinculados con la (in)suficiencia argumentativa que desembocan en una relación concesiva. Mientras que para Rodríguez Rosique la concesión aparece unida al uso del futuro, pues este tiempo implica un alejamiento por parte del hablante; Marcovecchio y Pacagnini parten de una estructura de finalidad en la que se produce también un cierto alejamiento, pero en este caso, de la finalidad hacia la concesión.

Para terminar, la estructura informativa y su dimensión discursiva también 
tienen su cabida en este monográfico a través del estudio que desarrolla Fernández Lorences. Esta autora analiza las diferencias y semejanzas entre tres tipos de estructuras: en relación con, por cierto y a propósito (de), especializadas en presentar el tema del que se habla o en introducir un cambio de tema en el discurso. El trabajo realiza un análisis en el plano gramatical, con el fin de definir el diferente grado de fijación de las estructuras mencionadas, pero también en el plano discursivo, puesto que se analiza su rendimiento en el discurso como unidades que intervienen en la estructura informativa.

El monográfico se completa con la reseña de Besa Camprubí al libro de Maria Josep Cuenca, Gramática del texto y con información acerca de la 7th Conference on Language, Discourse, and Cognition, que se celebrará en Taiwan, en mayo del 2014, así como de la revista Discours. Revue de linguistique, psycholinguistique et informatique.

\section{Conclusión}

Los trabajos reunidos tienen en común presentarnos el discurso como un tema de estudio que se redefine a partir de las últimas investigaciones. Las comunicaciones, paneles, proyectos que se realizan y que tienen como tema central el estudio del discurso en sus diferentes facetas es una muestra de este creciente interés. El monográfico que presentamos refleja el mismo interés.

Bibliografía

Asher, Nicolas y Alex Lascarides (2003): Logics of conversation. Cambridge: CUP. Garrido, Joaquín (2003): “Conexión y relevancia en la anáfora asociativa”, en José Luis Girón, F. Javier Herrero, Silvia Iglesia y Antonio Narbona (eds.), Estudios ofrecidos al profesor José Jesús de Bustos Tovar. Madrid: Editorial Complutense, pp. 115-124.

Garrido, Joaquín (2013): “Léxico y argumentación en la estructura del discurso”, en 
Carmen Llamas, Concepción Martínez Pasamar y Manuel Casado (eds.), Léxico y argumentación en el discurso público actual, (ed.), Léxico y argumentación. Lang: Francfort del Meno, pp.105-127.

Garrido, Joaquín y Teresa M. Rodríguez Ramalle (2013): "Relaciones de constituyentes en el discurso." VI Coloquio de Investigadores en Estudios del Discurso, ALEDar, Universidad de Quilmes (Argentina), 12-14 junio 2013.

González Ruiz, Ramón (2008): “Las nominalizaciones como estrategia de manipulación informativa en la noticia periodística: el caso de la anáfora conceptual”, en Inés Olza Moreno, Manuel Casado Velarde y Ramón González Ruiz (eds.), Actas del Actas del XXXVII Simposio Internacional de la Sociedad Española de Lingüística. Pamplona, Servicio de Publicaciones de la Universidad de Navarra.

Hidalgo Downing, Raquel (2003): La tematización en el español hablado. Madrid: Gredos.

Lascarides, A., Copestake, A. y Briscoe, T. (1996): «Ambiguity and coherence», Journal of Semantics, 13, pp. 41-66.

Llamas Saiz, Carmen (2010): “Argumentación en la noticia periodística: el caso de la anáfora conceptual metafórica”, en Concepción Martínez Pasamar (ed.), Estrategias argumentativas en el discurso periodístico. Frankfurt am Main: Peter Lang, pp. 147-170.

Mann, William y Sandra Thompson (1988): "Rhetorical Structure Theory: Toward a Functional Theory of Text Organization”, Text, 8 (3), pp.243-281.

Rodríguez Ramalle, Teresa M. (2008): "Estudio sintáctico y discursivo de algunas estructuras enunciativas y citativas del español”, Revista Española de Lingüística Aplicada, 21, pp. 269-288.

Smith, Carlota S. (2003): Modes of discourse. Cambridge: Cambridge University Press. Taboada, Maite (2006): “Discourse Markers as Signals (or Not) of Rhetorical Relations”, Journal of Pragmatics, 38(4), 567-592. 